\title{
LA ICTIOFAUNA DEL EMBALSE DE BEMPOSTA (RIO DUERO, PORTUGAL)
}

Alexandre C.N. VALENTE y Paulo J.B. ALEXANDRINO

Instituto de Zoologia "Dr. Augusto Nobre". facultade de Ciencias do Porto. Praça Gomes Teixeira - 4000 PORTO, PORTUGAL

Palabras clave: Embalse. Chondrostoma polylepis. Crecimiento.

\section{SUMMARY}

THE ICHTHYOFAUNA OF THE BEMPOSTA DAM (RIVER DUERO, PORTUGAL)

Key words: Dam. Chondrostoma polylepis. Growth.

We present the results of the fish population study conducted in the Bemposta dam (North Portugal).

A. list of the species capturated and the relative composition of the community are presented.

The iberian nase, chodrostoma polylepis STEINDACHNER, 1984, is the most important species. It was a good growth, in spite of the less favourable water characteristics (temperature and dissolved oxygen) that were observed during part of the year.

The carp, Cyprinus carpio LINNAEUS, 1758, and the iberian barbel, Barbus barbus bocagei STEINDACHNER, 1864, are less important species.

The available information on local fisheries is also refered.

\section{INTRODUCCION}

La presa de Bemposta es el tercer aprovechamiento hidroeléctrico del trozo internacional portugués del rio Duero (figura 1). Se localiza apro ximadamente a $280 \mathrm{Km}$ de la desembocadura y tiene una área de unas 430 Há.

La ictiofauna de nuestros cursos de agua no ha sido convenientemen te estudiada, por 10 que los estudios de este tipo son necesarios e importan tes para una adecuada ordenación pesquera. Dado el interés de la pesca profesional en este embalse, hemos tratado, también de evaluar la importancia local de ésta.

\section{MATERIAL Y METODOS}

Se realizaron dos muestreos en las inmediaciones de la presa, habiendose utilizado, al efecto, redes de pesca de varios tamaños de malla IV CONGRESO ESPAÑOL DE LIMNOLOGIA

Actas : $353-358$ 


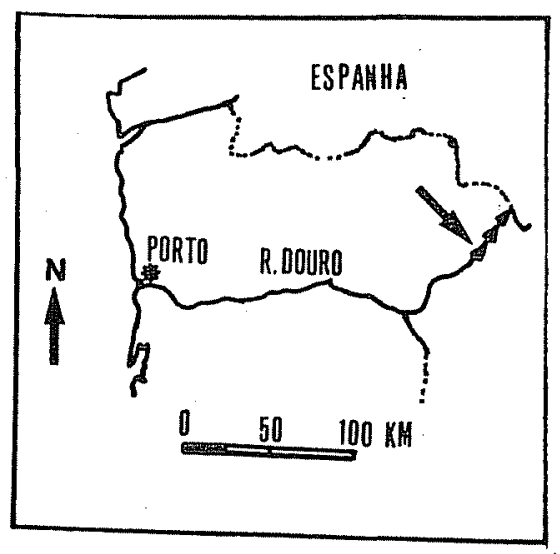

FIGURA 1

Mapa de situación del embalse de Bemposta.* Map of situation of the Bemposta reservoir.

$(18,20,22,24,26,28,30,35,40,55,57,60,65$ y $70 \mathrm{~mm})$. Los peces capturados fueron medidos y pesados $y$, de cada uno, se guardaron unas pocas escamas.

Para la determinación de la edad se recurrió al análisis de las escamas. Las correspondientes cada pez se lavaron y se montarón entre porta y cubreobjetos $y$ se observaron con un proyector de perfiles MITUTOYO PV350.

El cálculo de tamaño de los peces se hizo siguiendo la metodolo gía descrita por BAGENAL y TESH (1978). Las curvas de crecimiento se obtuvieron a través del modelo descrito por VON BERTALANFFY (en BAGENAL y TEȘH, 1978).

La relación peso/longitud, $\log w=\log a+b \log L$, se determinó por el método de los mínimos cuadrados.

El factor de condición se obtuvo por la ecuación descrita por FULTON (en BEGENAL y TESH, 1978), F.C. $=.100 \mathrm{~W} / \mathrm{L}^{3}$.

\section{RESULTADOS Y DISCUSION}

Las campañas realizadas han permitido establecer una lista de la ictiofauna local (cuadro 1) y su composición porcentual (Figura 2).

La boga de rio, Chordrostoma polylepis STAINDACHNER, 1865 es, sin duda, la especie más importante. Tiene un buen crecimiento (Figura 4) ya que, a pesar de las condiciones menos favorables del medio (figura 5), se asemeja a otros datos referidos a rios de la Peninsula Ibérica (LOBON-CERVIA, 1982; VALENTE, 1986). La relación peso/longitud (cuadro 2), muestra valores semejantes a los encontrados para otros rios europeos. La relación entre los sexos es nitidamente favorable a los machos ( $1: 2: 21$ ó), lo que puede ser resultado de un error de muestreo, ser indicador de condiciones desfavorables de reproducción (NIKLOSKII, 1969), o bién, ser el resultado de una diferénte distribución espacial de los sexos por motivos relacionados con fenómenos ligados a la reproducción (LOBON-CERVIA y ELVIRA, 1981).

La carpa, Cyprinus carpio LINNAEUS, 1758, es menos importante y la información de que disponemos es bastante mas escasa. La relación peso/ 


\begin{tabular}{|l|l|}
\hline \multicolumn{1}{|c|}{ ESPECIES } & NOMBRE VULGAR \\
\hline Chondrostoma polylepiss S. & boga de rio \\
Cyprims carpio L. & carpa \\
Barbus barbus bocagei S. & barbo \\
Leuciscus cephalus L. & escalo \\
Gobio gobio L. & göbio \\
Salmo trutta L. (1) & truta \\
Tinca tinca L. (1) & encala \\
Anguizla anguizla L. (2) & lücio \\
Esox Zucius L. (2) & \\
\hline
\end{tabular}

CUADRO 1

Lista de la ictiofauna del embalse de Bemposta, basada en la información propia, complementada por la de los pescadores locales. (1) Especie no capturada pero cuya presencia se confirmó por el exámen de ejem plares pescados en el embalse. (2) Especie no capturada pero que es pescada esporádicamente por los pescadores profesionales.

Check list of fishes of Bemposta reservoir. It is based on personal data complemented with information from local fishermen. (1) Non collected species. Specimens collected at Bemposta were examined. (2) Non collected speciss. It is occasionally reported by fishermen.

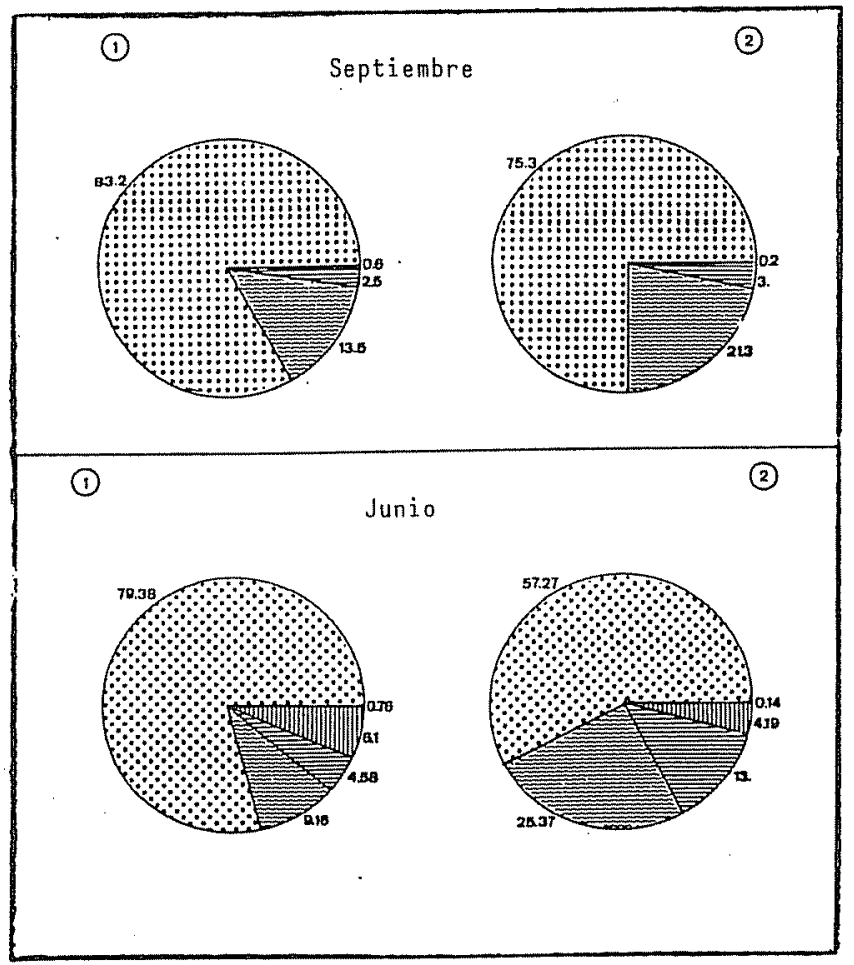

B boga

曰Escalo

圆昰的

圈 carps

FIGURE 2

Composición de las capturas realizadas en el embalse de Bemposta. (1) Abundancia re lativa de las especies. (2) Biomasa relativa de las especies.

D Bogs Composition of the captures 日 Gobio made in the Bemposta reservoir. (1) Relative abundance of species. (2) Relative aromas of the species 

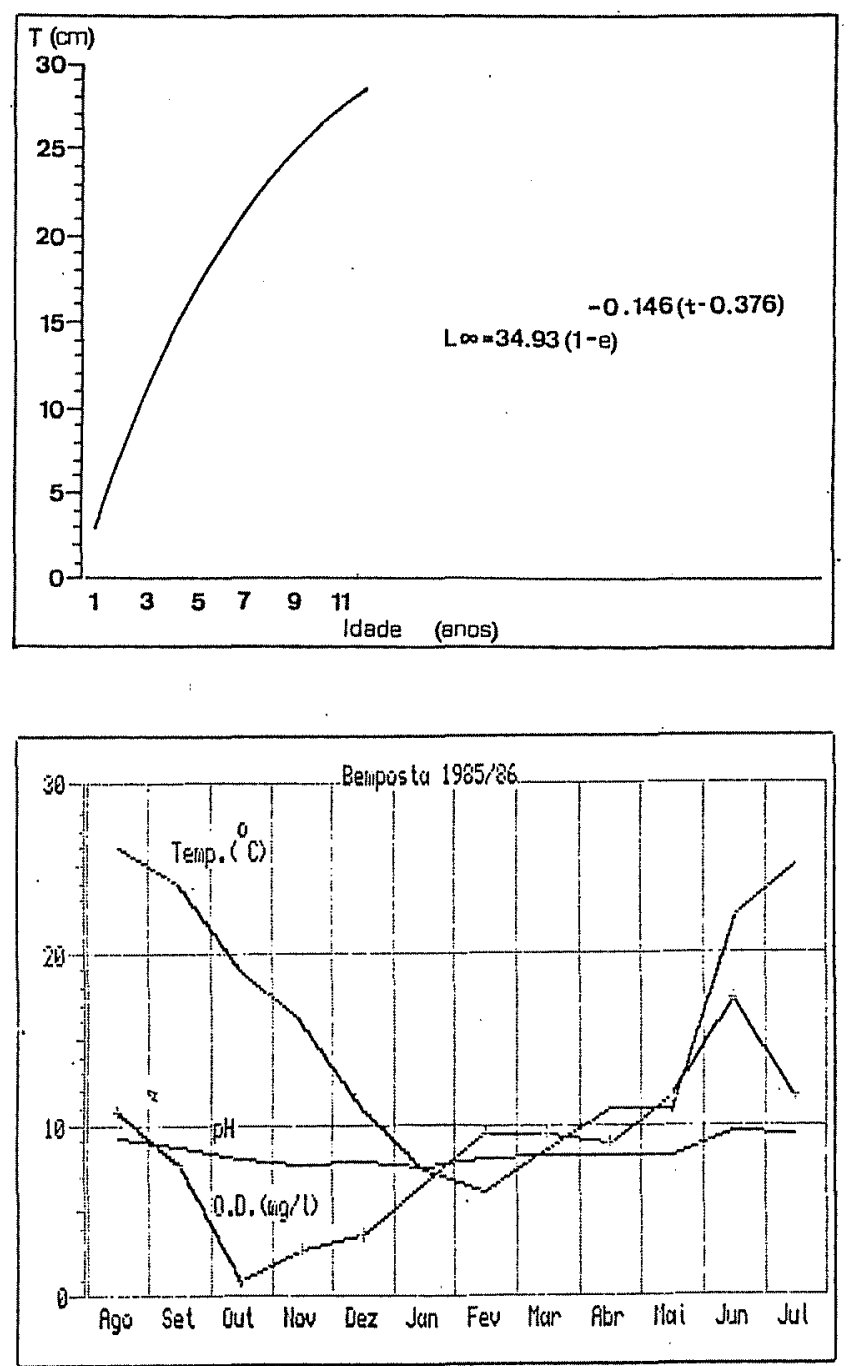

FIGURA 3

Curva teórica de crecimiento de Von Bertalanffy en las bo gas del embalse de Bemposta. Theoretical curve of growth of the nase of Bemposta reservoir.

FIGURE 4

Evolución de algunos parámetros fisico-químicos del embalse de Bemposta durante el periodo de estudio (CEQ/FEUP y IZUP/FCUP), 1986

Evolution of some physicoche mical. parameters of Bemposta reservoir during the study period (CEQ/FEUP and IZUP/FC UP), 1986.

entlongitud observada en ambos muestreos no difiere (cuadro 2). la relación entre los dos sexos es favorable a las hembras $(2 q: 106)$.

Las restantes especies son todavía menos importantes. Los barbos, Barbus barbus bocagei STEINDACHNER, 1864, pueden influir en el procentaje de la biomasa capturada (figura 2), una vez que alcanzan las mayores dimensiones del estado adulto. Se registran pocas capturas, pero los resultados observados (cuadro .2) deben ser considerados cuidadosamente. La relación entre sexos se determinó sólo en Junio ( 1 q : 2 ó).

La pesca profesional es ejercida por un reducido número de pescadores (unos 6) y sólo a tiempò parcial, alternativo a la actividad agrícola. Las declaraciones sobre capturas. realizadas por los pescadores son escasas y sin duda subestiman la pesca local. No obstante, permiten confirmar los datos obtenidos en nuestras campañas de muestreo en cuanto a la composición relativa de la comunidad y permiten apreciar la importancia económica de esta actividad (rendimiento bruto de 300 000\$00/mes/pescador - valor aproximado en los meses de actividad de pesca de los que tenemos registro). 


\begin{tabular}{|c|c|c|c|c|c|c|c|c|}
\hline ESPECIE & & LOCAL & EPOCA & a & b & $n$ & $r$ & \\
\hline Ch. polytepis & $\begin{array}{r}B . \\
R . \\
{ }^{R} B . \\
* *\end{array}$ & $\begin{array}{l}\text { Bemposta } \\
\text { Douro } \\
\text { Arrocampo }\end{array}$ & $\begin{array}{l}\text { Set. } \\
\text { Jun. } \\
- \\
- \\
-\end{array}$ & $\begin{array}{c}-1,47 \\
-1,63 \\
-1,- \\
-1,70 \\
1,9\end{array}$ & $\begin{array}{c}2,66 \\
2,79 \\
2,73-3,3 \\
2,80 \\
2,99\end{array}$ & $\begin{array}{r}10 \\
46 \\
- \\
82 \\
64\end{array}$ & $\begin{array}{c}0,99 \\
0,97 \\
-- \\
-- \\
--\end{array}$ & $\begin{array}{l}(1) \\
(1) \\
(2) \\
(3) \\
(3)\end{array}$ \\
\hline C. carpio & B. & Bemposta & $\begin{array}{l}\text { Set, } \\
\text { Jun. }\end{array}$ & $\begin{array}{l}-1,48 \\
-1,42\end{array}$ & $\begin{array}{l}2,80 \\
2,70\end{array}$ & $\begin{array}{l}11 \\
12\end{array}$ & $\begin{array}{l}0,99 \\
0,98\end{array}$ & $\begin{array}{l}(1) \\
(-1)\end{array}$ \\
\hline B. barbus & $\begin{array}{l}\text { B. } \\
\text { R. }\end{array}$ & $\begin{array}{l}\text { Bemposta } \\
\text { Jarama }\end{array}$ & $\begin{array}{l}\text { Jun. } \\
\text { Jun. }\end{array}$ & $\begin{array}{l}-1,66 \\
0,016\end{array}$ & $\begin{array}{l}2,89 \\
3,14\end{array}$ & $\begin{array}{r}6 \\
44\end{array}$ & 0,99 & (1) \\
\hline I. cephazus & $\begin{array}{l}B \text {, } \\
\text { R, }\end{array}$ & $\begin{array}{l}\text { Bemposta } \\
\text { Jarama }\end{array}$ & $\begin{array}{l}\text { Set. } \\
-\end{array}$ & $\begin{array}{l}-1,45 \\
-2,10\end{array}$ & $\begin{array}{l}2,69 \\
3,37\end{array}$ & $\begin{array}{l}8 \\
-\end{array}$ & $\begin{array}{l}0,98 \\
--\end{array}$ & $\begin{array}{l}(1) \\
(5)\end{array}$ \\
\hline
\end{tabular}

CUADRO 2

Parámetros de la ecuación de la relación peso/longitud $(\log W=\log a+b \log L$ ) para algunas de las especies encontradas en el embalse de Bemposta y en otros rios o embalses europeos.

$\mathrm{n}$ = tamaño de la muestra. $r=$ correlación. Datos de (1). presente trabajo, (2) CULEBBRAS y JALON, 1985, (3) GRANADO, 1979, (4) LOBON-CERVIA y FERNANDEZ-DELGADO, 1984, (5) LOBON-CERVIA y PENCZAK, 1984). (*) Machos, (**) Hembras.

Parameters of equation $\log W^{*}=\log a+b \log L$ (weight/length) of some species found at Bemposta reservoir and another european rivers or reservoirs.

$n=$ size of the sample. $r=$ correlation coefficient. Data from (1) This paper, (2) CULEBRAS Y JALON, 1985, (3) GRANADO, 1979, (4) LOBON-CERVIA Y FERNANDEZ DELGADO, 1984, (5) LOBONCERVia y PENCZAK, 1984. (*) Males, (**) Females.

\section{C.ONCLUSIONES}

L.as condiciones ambientales observadas en el embalse de Bemposta están próximas a los.límites de resistencia de los ciprínidos, aunque sólo durante un corto periodo del año. No obstante, son toleradas dada la gran capacidad de adaptación de estos peces (ALABASTER y LLOYD, 1980, HUGHES, 1981).

No se puede, todavía, extrapolar estas condiciones como si fuera lo que ocurre todos los años, ya que el crecimiento razonable de las formas presentes hace suponer situaciones más favorables.

Teniendo en cuenta el aprovechamiento local de la pesca, que tiene gran importancia, resulta del mayor interés preservar e, incluso, mejorar las condiciones que se han observado, de modo que estas potencialidades eco: nómicas no desaparezcan.

\section{BIBLIOGRAFIA}

ALABASTER, J.S., LLOYD, R., 1980.- Water quality criteria for freshwater fish. Butterworths. Londres.

BAGENAL, T.B., TESH, F.W., 1978.- Age and growth. En Bagenal, T.B. (ed.) Methods for the assessment of the fish production in freshwaters. I.B.P. Handbook ng 3. Blackwell Sci. Publ. Oxford. 
CEQ (FEUP) y IZUP (FCOP), 1986.- Estudo da qualidade da água da albufeira da Bemposta. Relatorio do trabalho para EDP.

CULEBRAS, E.B., JALON, D.G., 1985.- Edad y crecimiento de la boga de rio Chondrostoma polylepis, STEIN. 1865, en la cuenca del Duero. III Congreso Español de Limnol., Leon. 2-5 Julio. de 1985

GRANADO, C., 1979.- Estudio de las poblaciones de peces del embalse de Arrocampo (Cáceres). Tesis de Lic. Univ. Sevilla. En Lobon-Cervia, J., y Elvira B., 1981: Edad, crecimiento y reproducción de la boga de rio (Chondrostoma polylepis SETEIN. 1865) en el embalse de Pini Ila (rio Lozoya). Bol. Inst. Espa. Oceano., 6 : 200-213.

HUGHES, G.M., 1981.- Effects of the low oxygen and pollution on the respiratory system of fish. En Pickering, A.D., 1985.- Stress and fish. Academic Press. Londres.

LOBON-CERVIA, J., 1982.- Population analysis of the iberian nase (Chondrostoma polylepis STEIN. 1865) in the Jarama River. Vie et Milieu, $32(3): 139-148$.

LOBON-CERVIA, J., ELVIRA, B., 1981.- Edad, crecimiento y reproducción de la boga de rio (Chondrostoma polylepis STEIN. 1865) en el embalse de Pinilla (Rio Lozoya). Bol. Inst. Espa. Oceano., 6 : 200-213

LOBON-CERVIA J., FERNANDEZ-DELGADO, J., 1984.- On the biology of the barbel (Barbus barbus bocagei) in the Jarama river. Folia Zoologica, 33 (4) : 371-384

LOBON-CERVIA, J., PENCZAK, T., 1984.- Fish production in the Jarama river, central Spain. Hol. Ecol., 7 : 128-137

NIKOLSKII, G.V., 1969.- The theory of fish population dynamics as the biological background for rational exploitation and management of Fishe rie resources. Trad. de J.E.S. Bradley. Ed. por R.Jones. Moyler and Boyd, Ltd.

VALENTE, A.C.N., 1986.- As populaçoes piscícolas do rio Febros. 10 Congresso Inter. sobre o Rio Douro, V.N. de Gaia, 5/4 a 2/5 de 1986 . 